\author{
¿Cómo citar el artículo? \\ Rivera Sepúlveda, Á. A., y Guerra García, Y. M. (septiembre-diciembre, 2020). Tensiones y \\ discontinuidades en la comprensión comunitaria de la escuela rural. Revista Virtual Universidad \\ Católica del Norte, (61), 102-121. https://www.doi.org/10.35575/rvucn.n61a7
}

\title{
| Tensiones y discontinuidades en la comprensión comunitaria de la escuela rural
}

Tensions and discontinuities in the community comprehension of the rural school

\author{
Álvaro Andrés Rivera Sepúlveda \\ Doctor en Educación \\ Facultad de Educación y Humanidades, \\ Universidad Militar Nueva Granada \\ Bogotá, Colombia \\ alvaro.rivera@unimilitar.edu.co \\ Orcid: https://orcid.org/0000-0002-7405-7988 \\ Cvlac: http://scienti.colciencias.gov.co:8081/- \\ cvlac/visualizador/ge- \\ nerarCurriculoCv.do?cod_rh=0001449342
}

\author{
Yolanda Margaux Guerra García \\ Post Doctora en Narrativa y Ciencia \\ Facultad de Educación y Humanidades, \\ Universidad Militar Nueva Granada \\ Bogotá, Colombia \\ yolanda.guerra@unimilitar.edu.co \\ Orcid: https://orcid.org/0000-0002-7354-6798 \\ Cvlac: http://scienti.colciencias.gov.co:8081/c- \\ vlac/visualizador/ge- \\ nerarCurriculoCv.do?cod_rh $=0000184411$
}

Recibido: 25 de marzo de 2020

Evaluado: 30 de junio de 2020

Aprobado: 04 de septiembre de 2020

Tipo de artículo: Investigación Científica y Tecnológica.

\section{| Resumen}

La investigación indagó por las comprensiones locales sobre la escuela rural, a propósito de la hibridación entre el concepto de escuela modernizada y las concepciones sobre la escuela que pivotan en torno a la tradición de la comunidad. El trabajo de campo se adelantó en tres corregimientos rurales del municipio de Magangué, Colombia durante un periodo ininterrumpido de seis meses, desplegando un diseño multicíclico en tres fases, articulado a técnicas etnográficas: observación participante, entrevistas semiestructuradas y grupos de discusión. Los resultados evidencian cómo las mutaciones históricas, cruzadas por aspectos políticos principalmente, marcan profundas tensiones y discontinuidades en la manera como los actores rurales comprenden tres aspectos que son singularmente significativos para ellos: las relaciones escuela-comunidad, la figura del director escolar y la imagen del maestro. Se concluye que las comprensiones sobre la escuela que ostentan las comunidades rurales frecuentemente no se reconocen en las acciones de intervención que diseñan las instituciones públicas para mejorar el servicio educativo en este contexto, omisión que termina desatando relaciones antagónicas que desdibujan y problematizan el funcionamiento mismo de la escuela.

Palabras claves: Administrador de la educación; Educación rural; Investigación pedagógica; Relación escuela-comunidad; Situación del docente. 


\section{Abstract}

The research inquired about local understandings of rural school, about the hybridization between the concept of modernized school and the conceptions about school that revolve around the tradition of the community. The fieldwork was carried out in three rural districts of the municipality of Magangué, Colombia during an uninterrupted period of six months, deploying a multicyclic design in three phases, articulated to ethnographic techniques: participant observation, semi-structured interviews and discussion groups. The results show how historical mutations, mainly crossed by political aspects, mark deep tensions and discontinuities in the way rural actors understand three aspects that are uniquely significant to them: the relations between the school and the community, the figure of the school principal and the image of the teacher. It is concluded that the understandings about school held by rural communities are frequently not recognized in the intervention actions designed by public institutions to improve the educational in this context, an omission that ends up unleashing antagonistic relationships that blur and problematize the operation of the school.

Keywords: Education administrator; Rural education, Pedagogical research; School-community relationship; Teacher situation.

\section{A modo de introducción: mutaciones políticas en las comunidades rurales del municipio de Magangué}

Lo que se expone a continuación, a modo de introducción, es producto de las observaciones y diálogos in situ, en un intento por reconstruir etnográficamente las dimensiones social, cultural y política de lo que aún no se encuentra documentando ni escrito: la forma cómo las comunidades rurales del municipio de Magangué han experimentado el despertar de las instituciones democráticas y el balance que hacen de su evolución, especialmente en comparación con las privaciones materiales, pero también con los valores culturales que habían esculpido su experiencia comunitaria por décadas.

Durante la década de los años noventa las comunidades rurales del municipio de Magangué 1 comenzaron a experimentar un proceso de transición, a nivel comunitario, que los precipitó de un estilo de vida, caracterizado por la cooperación, la reciprocidad y el compromiso con los asuntos colectivos, a otro surcado por la indiferencia frente a los problemas comunes, el surgimiento de facciones sociales con intereses contrapuestos y la proliferación de prácticas clientelares de usufructo personal. Esta dislocación obedeció, principalmente, al despertar de las instituciones democráticas (elección de representantes locales, surgimiento de los procesos de descentralización e incremento de la inversión social) que tuvo lugar en esta región, durante los años noventa ${ }^{2}$ y que no sólo originó nuevas alteridades entre la administración pública local y las comunidades rurales, sino que abrió profundas grietas a nivel comunitario.

De acuerdo con la cosmovisión de los actores locales, en un principio, la fusión entre los contenidos de las instituciones democráticas y el repertorio axiológico de las comunidades favoreció el desarrollo de procesos públicos locales, como la construcción de centros de salud,

\footnotetext{
1 Magangué es uno de los municipios que componen al departamento de Bolívar; este último, ubicado en la región Caribe de Colombia.

2 Entre otros, autores como González y Liendo (2017) y Rodríguez (2017) identifican el final de los años ochenta y el inicio de los noventa como el periodo histórico de las transiciones a la democracia en América Latina.
} 
la modernización de las escuelas, el mejoramiento en la prestación de los servicios públicos sanitarios, la conformación de mecanismos de participación comunitaria, entre otros. Empero, en la medida en que dichas instituciones se fueron autonomizando frente a la cautela del Estado, empoderando cada vez más a la administración municipal, comenzaron a perpetuarse en el poder un acervo de elites locales que, con el decurso del tiempo, restaron valor a los programas sociales y se centraron exclusivamente en competir por el control del territorio. Es debido a circunstancias de este tipo que Rodríguez (2017) define la democracia como la historia de un desencantando y una indeterminación.

El anterior escenario ocasionó que los habitantes de las comunidades rurales ya no fueran prohijados como sujetos de derechos, sino como una nutrida masa electoral que podía ser captada mediante el intercambio de favores. En consecuencia, la institucionalidad democrática dejó de ser entendida como un vehículo para el desarrollo comunitario y se convirtió en una estrategia para compensar las necesidades y deseos en el plano personal. En efecto, Díaz y Paillama (2017) destacan cómo la participación en redes clientelares, soterradas en un aparente uso efectivo de la democracia, supone la solución a muchos problemas para personas que viven en contextos de pobreza permanente. En Magangué, concretamente, todas estas circunstancias alcanzaron su máxima expresión superado el umbral del 2001, cuando el municipio adquirió oficialmente la certificación administrativa ${ }^{3}$ y por esta vía confirió a las oligarquías locales mayor capacidad de maniobra para el despliegue de sus intereses; un fenómeno derivativo de los procesos de descentralización en América Latina que es problematizado por autores como Asela (2015) y Jiménez (2019).

Así, las nuevas coordenadas políticas no sólo originaron otras formas de relación entre las comunidades rurales y la administración municipal, sino que irrumpieron directamente en el entramado comunitario, imponiendo una nueva jerarquía de valores y provocando de esta manera el desgajamiento del repertorio axiológico endógeno, o lo que Saelzer et al. (2019) denominan valores culturales territoriales. Ciertamente, la lógica antagónica que permeó el advenimiento de las instituciones democráticas desencadenó un caudal de prácticas antitéticas con los valores que durante mucho tiempo les sirvieron a las comunidades para hacer frente a sus necesidades y expectativas comunes, esto es, su desarrollo comunitario de base (Herrera, 2016).

La cooperación para el trabajo colectivo fue remplazada por una actitud de total indiferencia frente a los problemas colectivos y una ausencia de compromiso frente al bienestar comunitario. Como alternativa, cada quien se vio impelido a buscar la forma de sobrevivir frente a las variadas privaciones, de acuerdo con sus propios medios, asi como usurpando el disfrute colectivo de los bienes públicos para responder a los deseos y necesidades del ámbito doméstico; esto sin mucha empatía frente a las necesidades de las otras personas y con muy poca conciencia sobre el profundo daño que se le estaba ocasionando a la comunidad en general. Precisamente a este hecho se refiere Martínez (2019) cuando sostiene que los mecanismos clientelares se han convertido en un obstáculo para la consolidación de la organización social en el medio rural.

3 De conformidad con la Ley 715 de 2001, los municipios certificados gozan de autonomía para administrar los recursos que les transfiere el Estado, y así financiar los servicios a su cargo, en tres aspectos principalmente: educación, salud y saneamiento básico. 
La reciprocidad entre individuos, por su parte, fue desplazada por una actitud de profunda desconfianza frente a las intenciones de las otras personas, socavando de esta manera las relaciones interpersonales de parentesco y de vecindad. De hecho, se pudo constatar una cosmovisión generalizada de suspicacia frente a cualquier iniciativa que pretende hilvanar la organización de la población para la obtención de beneficios comunes. Según se pudo constatar, siempre que alguna persona pretende impulsar una iniciativa comunitaria, automáticamente sus buenas intenciones son desacreditadas por los demás miembros de la comunidad, con especulaciones acerca de sus verdaderos propósitos, consiguiendo que no obtenga ningún tipo de adhesión y que finalmente se decante por desmarcarse de los procesos sociales. Esta situación inquieta, aún más, si se reconoce que, en contextos locales como este, las acciones colectivas, a diferencia de las de carácter público y privado, son las que más contribuyen a la gestión del territorio (Sili, 2019).

Entretanto, la figura del líder comunitario, que otrora encarnó un rol fundamental para impulsar el desarrollo endógeno de las comunidades, degeneró en un mediador para el intercambio de favores entre los dirigentes políticos y los pobladores rurales. Hoy en día, se denomina líder a quien detenta el poder administrativo sobre alguno de los bienes sociales de la comunidad y aglutina entorno a sí a cierto número de personas, conformando con ellos un grupo o facción social, que opera exclusivamente para favorecer los intereses de sus miembros, erosionando de esta manera el crecimiento y desarrollo de toda la comunidad. Son miembros que, como lo sostiene Martínez (2019), terminan idealizando su relación clientelar con el denominado líder o patrón, debido a la dinámica del don y contradon que esta representa. Como resultado, entre el grueso de la población, hoy nadie declara aquiescencia por los liderazgos ni por las buenas intenciones de quienes pretenden impulsar alternativas de transformación.

Por consiguiente, la organización de la comunidad como un todo integrado con objetivos comunes ha sido absorbida por el surgimiento de subgrupos con intereses contrapuestos que compiten entre sí por satisfacer sus propias necesidades. En efecto, Codoceo y Muñoz (2017) sostienen que las instituciones públicas en una democrática neoliberal, al enfocarse más en resolver la situación material individual que en fomentar valores como la solidaridad y la confianza social, resultan siendo marcadamente disgregadoras y fragmentarias. En este sentido, se pudo apreciar en cada pueblo la coexistencia de cinco a ocho facciones claramente diferenciadas, cuya conformación, en armonía con Durston et al. (2016), tuvo un origen espontáneo en las rivalidades entre familiares y sectores, pero que con el decurso del tiempo se han ido sedimentando gracias a las coaliciones clientelares con los patrones políticos municipales. Dentro de las facciones identificadas se encuentra el centro de salud, la escuela, la iglesia, la agremiación de madres comunitarias, la junta de acción comunal, la comercializadora de leche, el comité del acueducto y la asociación de pescadores. Según las diferentes voces, esta atomización ha conducido a un conflicto interno que no sólo enquista la cohesión de la comunidad, sino que obstruye la posibilidad de hacer frente a los problemas comunes.

Según se pudo reconstruir, el modus operandi de las diferentes facciones se conjuga alrededor de cinco prácticas concretas que dan cuenta de la complejidad de los conflictos: i. tomar decisiones sobre el bien social que administran, sin contar con el concurso de la comunidad; ii. desacreditar y entorpecer las iniciativas de los otros grupos; iii. vulgarizar los logros obtenidos para ostentar un mejor nivel de vida frente a los demás; iv. apropiarse de las ayudas que se envían desde la administración municipal, impidiendo su disfrute por parte de los grupos opuestos, lo que apunta a criterios de merecimiento y desmerecimiento constitutivos de la relación clientelar en la distribución de beneficios y recursos (Díaz y Paillama, 2017); y v. captar el mayor volumen de masa electoral para garantizar que el portavoz político con el que sostienen relaciones se mantenga en el poder y los retribuya con toda clase de prebendas. 
A instancia de todo lo que antecede, los actores locales analizan que el despertar y devenir de las instituciones democráticas, después de 30 años de funcionamiento, si bien abraza un aparente fortalecimiento en términos formales, no ha contribuido a resolver las variadas limitaciones sociales existentes en las comunidades, y tampoco ha impulsado la participación del campesinado en la concertación de alternativas, lo cual sintoniza con los planteamientos del Pérez-Liñán y Mainwaring (2015) y Braga y Acuña (2015), a propósito del estado de la democracia en América Latina. En oposición, ha servido exclusivamente para desmoronar el repertorio axiológico endógeno, sumergiendo a las comunidades en una cultura política caracterizada por la prioridad de los intereses personales, las escisiones internas y el intercambio de favores.

En el marco de este panorama, el objetivo del presente trabajo es mostrar cómo la evolución de las instituciones democráticas en Magangué, además de provocar nuevas dinámicas comunitarias en las áreas rurales, también han originado una suerte de hibridaciones entre el modelo de escuela rural que ellas privilegian (modernizada) y las concepciones sobre la escuela rural que pivotan en torno a la tradición de la comunidad. En concreto, se describen las tensiones y discontinuidades que se están produciendo en la comprensión de la escuela por parte de los actores rurales, particularmente en tres aspectos: las relaciones escuela-comunidad, la figura del director escolar y la imagen del maestro.

\section{| Método: hacer etnografía escolar}

El municipio de Magangué, ubicado en el departamento de Bolívar, está conformado por una cabecera municipal y cerca de 30 corregimientos rurales, en su gran mayoría localizados a orillas del río Magdalena. El trabajo de campo tuvo lugar en tres de estos corregimientos: La Pascual, Tacaloa y Coyongal (ver figura 1), durante los meses de julio y diciembre de 2017; cuya selección 冈en asesoría con autoridades locales $\bigotimes$ obedeció a un único criterio: aquellos que representaban la heterogeneidad del municipio en aspectos geográficos (grado de dispersión y tipo de cultura según cercanía con el río), productivos (actividad económica principal) y sociales (satisfacción de servicios públicos básicos).

\section{Figura 1}

Ubicación de La Pascual, Tacaloa y Coyongal en el municipio de Magangué

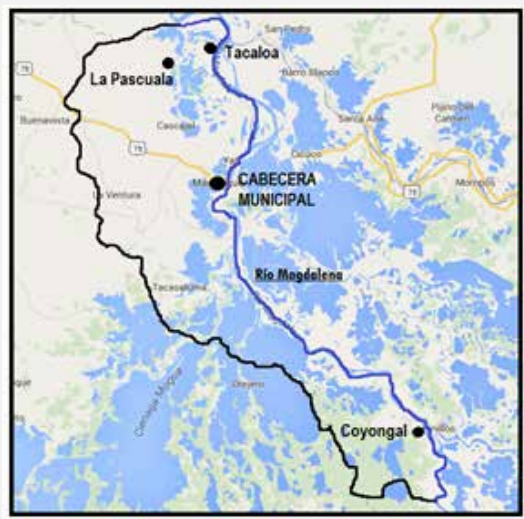

Nota: adaptado de Google Maps. 
El acceso a las comunidades se gestionó exponiendo abiertamente las implicaciones de la investigación en la Secretaria de Educación municipal, instancia que aprobó la iniciativa y posteriormente favoreció los canales de comunicación con los directores escolares de cada institución educativa. Surtido este trámite, y una vez sobre el terreno, se acordó en cada institución desempeñar el rol de maestro visitante, conviviendo en el hogar de una familia campesina y asistiendo todos los días al centro educativo, según las costumbres del lugar. Las primeras semanas in situ se dedicaron exclusivamente a explorar las condiciones objetivas del sector: socioculturales, económicas y políticas, así como a construir alteridades de empatía con los diferentes actores. De modo que, además de cumplir con los compromisos como maestro, también se asistió a reuniones de la junta de acción comunal, se participó en eventos culturales, religiosos y deportivos, y se dialogó con personas no necesariamente vinculadas al centro educativo, como líderes comunitarios, campesinos y algunos comerciantes.

Esta primera fase contribuyó, por una parte, a reconocer la idiosincrasia del lugar y, por esta vía, a entender la conexión de la escuela con lo que Ogbu (2005) denomina otras fuerzas sociales existentes; a su vez, sirvió para comprender que las concepciones sobre la escuela, que ostentan los sujetos, están configuradas a partir de las estructuras sociales y políticas a nivel macro, y de ninguna manera constituidas como construcción, sustraídas de estos marcos (Guber, 2016; Sautu, 2015). Por otra parte, coadyuvó a decantar las primeras hipótesis de trabajo, así como a depurar las categorías de indagación previamente bosquejadas desde los referentes teóricos de la investigación (Hammersley, 2016; Sautu, 2015).

Con un panorama más diáfano sobre las condiciones del lugar y un terreno abonado en la construcción de alteridades, se procedió a programar entrevistas individuales semiestructuradas con diferentes informantes dentro de cada grupo de actores; para esto fue necesario hacer la petición formal a cada persona y posteriormente coordinar con ellos la fecha y lugar del encuentro según su disponibilidad. En total, se dialogó con once estudiantes de los grados superiores, dieciséis maestros, tres directivos docentes, seis padres de familia y tres exalumnos. Esta fase del trabajo de campo estuvo enfocada en interpretar el mapa de significaciones de los sujetos, escudriñando en sus concepciones sobre la escuela, a partir de los hechos observados y cotejando los diferentes testimonios entre sí, dando lugar a microciclos de indagación, integrados por el descubrimiento de categorías emergentes, la formulación de nuevas hipótesis de trabajo y el desarrollo de estas por vía de diálogos cada vez más afinados. Es por eso que en la mayoría de los casos se desplegó una secuencia paralela de conversaciones en dos y tres sesiones.

Una de las ventajas en la selección del método etnográfico fue la posibilidad de hablar con los sujetos, no a partir de supuestos teóricos exclusivamente, sino en función de los hechos presenciados, las intuiciones propias sobre los mismos y la permanente confrontación de las diferentes perspectivas entre sí, todo lo cual contribuyó a que los actores también se implicarán en un proceso de auto-interpretación y auto-comprensión de su propio entramado de significados (Geertz, 1992). Además, favoreció el despeje de algunas visiones sobre la escuela, excesivamente marcadas por las rivalidades entre grupos y los conflictos de intereses propias del sector.

La tercera fase del trabajo de campo consistió en la resignificación de las primeras interpretaciones por vía de la intersubjetividad y de la construcción de consensos (Ibáñez, 1992). Fue así como se procedió a desarrollar un total de nueve grupos de discusión: tres con estudiantes, tres con maestros y tres con miembros de la comunidad, todos orientados a profundizar en el análisis de algunas categorías que, de acuerdo con el corpus disponible, se perfilaban como construcciones intersubjetivas de sentido común sobre la escuela en el lugar (Schütz, 1995); en concreto: las relaciones escuela-comunidad, la figura del director escolar y la imagen del maestro. Todos los grupos se conformaron con personas que previamente habian participado en las entrevistas individuales, esto para mantener el hilo conductor de las conversaciones y de lo hallado en la primera etapa del diseño. 
Cabe resaltar, además, que en la tercera fase fue necesario precisar algunos tópicos por medio de la realización de nuevas entrevistas individuales, por lo que se trató más de un proceso multicíclico que lineal (Wolcott, 2016). Igualmente, y en simultaneidad con los análisis in situ, en un viaje de ida y regreso hasta el final del estudio, se adelantó un procesamiento sobre el escritorio que consistió en la codificación y categorización del corpus progresivamente disponible, producto de lo cual fueron emergiendo y tomando forma como categorías centrales los tres aspectos que se desarrollan en el acápite de resultados.

Siendo así, las comprensiones locales sobre la escuela que se exponen a continuación no son el resultado de una recolección de información apresurada y un análisis de contenido monológico por parte del investigador, sino el producto de un itinerario etnográfico en el que el reconocimiento de las condiciones objetivas del lugar operó como marco de posibilidad para comprender la concepciones subjetivas e intersubjetivas de los propios sujetos; itinerario en el que los actores locales se vincularon no en calidad de meros respondientes, sino como productores de sentido, y por eso mismo artífices en el proceso de análisis e interpretación de su propia realidad social y educativa.

\section{| Resultados}

En coherencia con lo anunciado en el acápite introductorio, los resultados que siguen muestran en qué consisten las tensiones y discontinuidades que experimentan los actores rurales en su manera de comprender tres aspectos de la escuela, que para ellos en particular resultan significativos: la relación escuela-comunidad, la figura del director escolar y la imagen del maestro. Los relatos empíricos citados se encuentran codificados teniendo en cuenta tres tipos de datos: i. el estamento, Ma (maestros), Di (directivos), Es (estudiantes), Pf (padres de familia); ii. el corregimiento, Pa (Pascuala), Ta (Tacaloa), y Co (Coyongal); y iii. la técnica, Ei (entrevista individual), Gd (grupo de discusión).

\section{Comprensiones sobre las relaciones escuela-comunidad}

El surgimiento de las escuelas rurales del municipio de Magangué es una respuesta de las mismas comunidades a la ausencia de establecimientos escolares para los niños y jóvenes del medio; es por eso que, en su gran mayoría, todas ellas fueron construidas y sostenidas durante sus primeros años de funcionamiento por la propia población. No sorprende entonces que desde su fundación y hasta los primeros años del presente siglo, estos centros operaran en medio de circunstancias materiales adversas: su planta física se encontraba en malas condiciones, los recursos eran prácticamente inexistentes, no se ofrecían todos los niveles escolares, el índice de cobertura era muy bajo y existían muy pocos maestros.

Se trataba de "pequeñas escuelitas", dispersas sobre la geografía del municipio, que operaban en predios frecuentemente prestados o donados por alguna de las familias del pueblo, y cuya edificación se limitaba a una única aula en la que un solo docente (quien hacía las veces de enseñante y coordinador de manera simultánea) impartía todos los niveles de la básica primaria al mismo tiempo (escuela multigrado). La comunidad facilitaba las condiciones para la estadía del docente (cuando era necesario) y se organizaba voluntariamente para atender las necesidades apremiantes que las instalaciones precisaban. 
Sin embargo, la obtención de la certificación administrativa del municipio en 2001 y, por esta vía, la canalización directa de recursos del Sistema General de Participaciones (SGP), condujo a que la educación rural comenzara a experimentar, de manera progresiva, mayor inversión y reglamentación en el plano administrativo: la planta física de algunas escuelas fue ampliada y mejorada, comenzaron a llegar recursos pedagógicos no antes vistos, como equipos informáticos y conexión a internet; se oficializó el agrupamiento de escuelas, se designaron directores escolares con facultades administrativas, se reglamentó e incrementó el nombramiento de maestros, se aumentaron los índices de cobertura y se concedió a cada centro su propio presupuesto.

Así, se produjo una evolución del modelo de escuela tradicional, signado por un evidente abandono de la mano del Estado, al modelo de escuela modernizada, identificado por una mejor dotación de recursos humanos y materiales, así como por una oferta más amplia de programas pedagógicos diferenciados 4 . No obstante, los propios actores rurales reconocen que, si bien este hecho condujo a una optimización material del servicio educativo y coadyuvó a elevar los índices de cobertura, también fue en detrimento tanto de su función social como de algunos valores que la escuela tradicional había privilegiado y con los cuales ellos parecían identificarse, provocando de esta manera hibridaciones entre los dos modelos y, por esta vía, discontinuidades en su forma de comprensión. En consonancia con Juárez y Rodríguez (2016), si bien en las últimas décadas se han incrementado los niveles de acceso de la población rural al sistema educativo, subsiste el hecho de que esta estrategia por si sola se queda corta en la aspiración de ofrecer una educación integral y de mejor calidad para esta población.

En este caso en particular, los actores valoran otros aspectos más allá de la sola cobertura; uno de los más destacados para ellos es el nexo escuela-comunidad. Según su perspectiva, y en continuidad con lo expuesto por Castro (2007), consideran que, en zonas rurales alejadas de los centros urbanos, las relaciones entre las dos partes deben ostentar altos niveles de interdependencia y reciprocidad; de hecho, así lo experimentaron desde el surgimiento mismo de los centros: la población trabajaba en el sostenimiento de la escuela (porque era necesario), al tiempo que la escuela lideraba iniciativas de organización comunitaria (porque era importante). Al respecto, una maestra relata:

Realmente, en alguna ocasión ejecutamos proyectos de impacto en la comunidad, por ejemplo, sacar las calles, porque aquí había sólo una calle, entonces desde el colegio lideramos una campaña para sacar otra calle, reunimos a la comunidad, tumbamos monte y lo hicimos; muchos de estos salones los construyó la comunidad, porque entre maestros y estudiantes vimos la necesidad y convocamos a la comunidad, también hicimos arborización en las orillas del río, eso lo hicimos nosotros. (Ma-Ta-Gd)

En el relato de esta maestra se observa cómo desde la escuela se lideraban iniciativas sociales, de diferente naturaleza, que favorecían la resolución de problemas a nivel comunitario; sin embargo, en la medida en que las escuelas encararon el proceso de modernización por vía de la institucionalidad democrática, las relaciones de reciprocidad con la comunidad comenzaron a cercenarse. Según la óptica local, el incremento de recursos escolares, especialmente a través del Fondo de Servicios Educativos (FOSE), y las facultades que se les confirieron a los directores para administrarlo, originaron una suerte de antagonismos culturales entre la comunidad y la institución; en términos de Wolcott (2005), una superposición de nuevos valores y erosión de las costumbres del lugar.

4 Por alguna razón que valdría la pena explorar, la década de los años noventa representa para Iberoamérica un punto de inflexión en la implementación (o consolidación) de programas diferenciados y flexibles para el mejoramiento de la educación en el medio rural. Así lo documentan Juárez y Rodriguez (2016) para el caso mexicano; Mayoral et al. (2018) para el caso español, y Ortega y Cárcamo (2018) para el caso chileno. 
En efecto, en comunidades rurales donde los recursos son escasos y las fuentes de empleo prácticamente inexistentes, el arribo del presupuesto escolar y junto con ello la oferta de contratos laborales, originó que la escuela pasara de ser un escenario que precisaba de la solidaridad comunitaria para su sostenimiento a una fuente inagotable de empleo y de recursos. "El colegio de aquí es un negocio, esta institución es una mina, así se puede ver" (PF-Co-Ei), alude uno de los miembros de la comunidad, dilucidando tensiones en su forma de comprender hoy al centro educativo.

En consecuencia, un segmento importante de la población comenzó a desafectarse de los procesos escolares, puesto que, como se pudo conocer, para muchas personas ya no tiene ningún sentido esforzarse en trabajar por el sostenimiento de la escuela bajo el argumento de que ahora existe un presupuesto escolar con el cual se pueden satisfacer todas las necesidades. La formación de esta concepción entre la gente es aún más problemática si se tiene en cuenta que, de los cuatro frentes que contempla la Ley 715 de 2001 en lo que se refiere a las participaciones con destinación al sector educativo (nómina, infraestructura, canasta y calidad educativa), más del 75 \% se emplea exclusivamente en el salario del personal docente y administrativo (Ariza et al., 2017), por lo que la implicación de la comunidad en el buen funcionamiento de los centros (infraestructura, dotación de recursos, realización de actividades) sigue siendo una necesidad.

Adicionalmente, la población ha venido experimentado una sensación de desconfianza frente a la transparencia con la cual se administran los recursos, especialmente porque, según ellos, los fondos asignados a la institución educativa son abundantes y aun así el estado de algunas escuelas no mejora en proporción, esto en armonía con lo hallado por Zúñiga y Valencia (2016) en un estudio similar adelantado en la ciudad de Cartagena. Dichas circunstancias han suscitado entre los diferentes individuos una reacción de resentimiento y desconfianza frente a los directores y demás funcionarios escolares, al punto de provocar serias escisiones entre la comunidad y la escuela como institución. "La población tiene la percepción de que los funcionarios escolares vienen con la intención de usurpar los recursos educativos y aprovecharse de la hospitalidad de las personas, mas no para ayudarlos a salir adelante" (Di-Ta-Ei), señala uno de los maestros.

Por lo anterior, la figura del director escolar ha adquirido una jerarquía especial en el interior de las comunidades, derivando en un personaje que ostenta recursos y poder, dando origen a una suerte de relaciones clientelares endógenas o clientelismo autoritario provinciano (Durston et al., 2016), en cuyos términos el director concede la exclusividad de los contratos escolares a un recorte de personas, a cambio de su apoyo en la toma de decisiones y de su respaldo frente a las inconformidades manifestadas por el grueso de la población.

Nosotras no tenemos derecho a tener un trabajo en el colegio, porque como no estamos por la corriente del rector, entonces sólo los de esa corriente tienen derecho a tener un trabajo, eso es lo que está pasando aquí en esta comunidad. Aquí el que quiere conseguir un puesto de trabajo en el colegio tiene que conseguir unos votos y estar en la rosca del rector. Pero uno no puede decir nada malo del rector, porque se echa a la comunidad encima, la gente a la cual él rector favorece lo defiende. Pero nosotros como padres de familia también deberíamos tener la oportunidad de trabajar en el restaurante o en el aseo del colegio, pero nunca podemos, porque siempre son los mismos y los mismos. (PF-Pa-Gd)

Es así como la escuela se ha convertido en un escenario de conflictos y rivalidades entre grupos con intereses en colisión, que no sólo está erosionando las relaciones entre la escuela y la comunidad, sino que ha contribuido a reproducir la fragmentación social y a recrudecer las enemistades entre familiares y vecinos, lo que Machado (2017) describe como un fenómeno idiosincrático de los sectores rurales en Colombia. Como lo deja entrever el anterior testimonio, el recorte de individuos que se beneficia de su filiación con el director escolar es frecuentemente puesto en tela de juicio por quienes se encuentran al margen y también exigen oportunidades. 
Con todo, la población es consciente de que el problema de fondo no radica en el incremento del presupuesto público y mucho menos en que las escuelas se hayan modernizado, de hecho, valoran este avance como algo necesario; el problema para ellos estriba en la forma como se están administrando los recursos y las reacciones que esto suscita entre los diferentes pobladores, de suerte que la escuela ya no está fungiendo como una institución social que favorece la cohesión y organización comunitaria (lo que sí lograba la escuela tradicional a pesar de sus limitaciones materiales), sino como una instancia que cada vez más recrudece los conflictos ente diferentes segmentos de la población.

Según su óptica, se podrían desplegar algunas acciones concretas que coadyuven a que la población recupere la confianza en la escuela y se encuentre mucho más dispuesta a participar de sus procesos, subsanando de esta manera las relaciones entre las dos partes y recuperando el lugar social que los centros escolares han de cumplir en medio de las comunidades, como: incluir a la población en la elaboración del presupuesto escolar, rendir periódicamente un informe transparente sobre el estado de las cuentas y rotar los contratos de trabajo, de modo que todas las familias disfruten de oportunidades.

\section{Comprensiones sobre la figura del director escolar}

Existe consenso entre los diferentes actores respecto a que un director escolar en el medio rural debe comportarse como un auténtico líder comunitario, con alto sentido de pertenencia por los asuntos de la comunidad, capacidad de empatía frente a las necesidades de la población y un carisma especial en el trato con la gente. Es así, porque siendo la escuela la única institución social que funciona de continuo y sus funcionarios los únicos sujetos cualificados, el director encarna un protagonismo particular que trasciende las paredes del centro educativo y se incardina en el entramado social más amplio. Ciertamente, la percepción de la población sobre la figura del director escolar alude a prácticas como: visitar los hogares de las familias para conocer más de cerca sus necesidades, interactuar con la gente en diferentes escenarios y no sólo en su despacho, convocar a la comunidad en general para trabajar de manera articulada en la resolución de los problemas comunes, orientar los procesos escolares en dirección a la comunidad y estimular el liderazgo comunitario entre maestros y estudiantes.

No obstante, esta manera de significar el papel de los directores escolares parece entrar en permanente tensión respecto a la forma como los actuales funcionarios que ocupan este cargo se están desempeñando. Según se pudo advertir, el nombramiento de directores escolares provenientes de otras regiones, aunado a la total autonomía de la que estos disfrutan para regentear los recursos del establecimiento educativo, ha conducido a que muchos de ellos se centren exclusivamente en la dimensión administrativa de su función, manifestando escaso interés por los problemas sociales que experimenta la población, razón por la cual no es extraño que zanjen malas relaciones con los padres de familia y con la comunidad en general.

De acuerdo con las diferentes voces, los directores escolares parecen más preocupados por la administración de los recursos que por hilvanar iniciativas en el plano pedagógico y comunitario. En su mayoría, no son oriundos del pueblo donde se encuentra ubicada la escuela, de modo que llegan en la mañana y se marchan una vez finaliza la jornada, y, en ocasiones, asisten a la escuela, únicamente, dos o tres días por semana, ejerciendo una dirección en la distancia. Aunque toda la comunidad reconoce quién es el director de la escuela, lo conciben como un personaje ajeno que exhibe un prurito de superioridad. La comparación que propone una madre y maestra sirve para ilustrar este planteamiento: 
Yo recuerdo la primera rectora que tuvimos, era una líder que tenía un carisma, ella llamaba a la gente, al que no iba lo visitaba, iba a nuestros hogares, conversaba y mostraba lo bueno, ese es el primer líder que es el jefe. Pero esa parte se perdió, ese liderazgo por parte del jefe; ya él no va al hogar, ya él no va a visitar para entusiasmar a la gente para que participe, sino que yo vengo y me siento aquí en el pódium y todo el mundo tiene que llegar a mí, entonces él está en esa posición, los papás estamos en la misma posición "si él no viene acá, yo tampoco voy para allá", y lo jóvenes también se achantan. (Pf-Co-Gd)

En la segunda parte de este testimonio, la maestra hace énfasis en cómo el cambio de enfoque de los directores actuales ha desatado también un cambio de perspectiva por parte de la comunidad en general, dando lugar a un ciclo vicioso que lesiona los principios de la democracia escolar. Efectivamente, se pudo observar que los directores escolares evidencian un alto grado de escepticismo frente a la participación de las familias en el establecimiento educativo; frecuentemente buscan la manera de que los padres de familia no se involucren demasiado en los asuntos escolares o de que se autoexilien por falta de apoyo a sus iniciativas, procurando de esta manera detentar el control absoluto sobre todos los procesos, hecho que conviene con los planteamientos de Díaz (2016) acerca de los límites a la democracia y la participación escolar.

En las tres escuelas visitadas los padres de familia confesaron sentirse cada vez más marginados del centro educativo, especialmente en lo que se refiere a la discusión de temas cardinales para el buen funcionamiento de la institución. "Cuando yo fui miembro del consejo directivo, nos llamaban para instalarlo, yo firmaba como representante de los padres, pero jamás nos volvían a citar y tampoco sabíamos qué hacía con las cosas, de esa manera el colegio nos vincula" (PF-Co-Gd). Es precisamente esta forma de operar -a puerta cerrada y de espaldas a la gente- la que ha originado entre la población campesina sentimientos de recelo e indignación que recrudecen los antagonismos. Lo anterior, en contravía con lo expuesto por el Ministerio de Educación Nacional -MEN- (2017), cuando asegura que, para conseguir mayor coherencia entre la oferta educativa de la escuela y las necesidades del medio rural, se hace imperativo apalancar el concurso de las comunidades locales en los procesos de planificación e implementación de los proyectos escolares.

Igualmente, se pudo evidenciar entre los directores escolares pocas iniciativas en la realización de proyectos pedagógicos que tengan impacto en la comunidad, así como en la ejecución de trabajos sociales que respondan a las problemáticas locales, incluso, suelen desestimar las alternativas de trabajo comunitario que proponen los estudiantes y los mismos maestros, argumentando la inexistencia de tiempo y de recursos. De acuerdo con algunos testimonios, tradiciones culturales como la celebración de las fiestas patronales y otras afines se han ido disipando debido a la falta de iniciativas por parte de quienes dirigen los centros escolares.

Así las cosas, el vaciamiento del liderazgo comunitario entre los directores escolares se ha convertido en una de las razones que está incidiendo, de manera notable, en que la escuela no pueda entretejer relaciones de mayor armonía con la comunidad. En línea con Ortega y Cárcamo (2018), se puede asegurar que "el tipo de liderazgo se configura como pieza fundamental para motivar y facilitar la participación de las familias en la escuela, así como también para el fortalecimiento de la relación entre ambos agentes" (p. 109). La población es consciente de que la escuela disfruta de todo el potencial para dinamizar procesos sociales en el interior de las comunidades; de hecho, reconocen que esta tarea hace parte de sus funciones esenciales, empero, advierten que para agenciarlo se requiere un cambio de enfoque por parte de quienes vienen a regentar las instituciones educativas 
Proyectando lugares de distensión entre sus concepciones y las circunstancias actuales, los diferentes actores reconocen que los directores escolares no se deben limitar a la administración de la escuela como un servicio que sirve exclusivamente a la escolarización; más allá, es imprescindible que ostenten una implicación activa en la vida de las comunidades y una vocación especial para bordar los hilos de la escuela, de tal forma que contribuyan a tejer otros paisajes a nivel comunitario. Como lo destacan Barrientos et al. (2016) es fundamental que los directivos movilicen habilidades, cualidades y valores personales para asegurar mayor articulación con la comunidad. En consecuencia, el perfil de director escolar en este contexto debe abrazar otras facultades, como sensibilidad social y empatía con las necesidades de la población, capacidad de liderazgo, disposición para trabajar de la mano con la gente y conocimiento de la idiosincrasia del sector.

\section{Comprensiones sobre la imagen del maestro}

De acuerdo con las voces de los diferentes actores, los maestros que se desempeñan en el medio rural, a diferencia de sus homónimos urbanos, no se deben confinar exclusivamente al cumplimiento de sus responsabilidades académicas dentro del aula, por el contrario, deben trascender su quehacer de modo que abrace un impacto directo en el entorno. Aseguran que los maestros disfrutan de condiciones propicias (formación, conocimientos y credibilidad) para liderar procesos públicos que contribuyan a apalancar el desarrollo de las comunidades, en lo fundamental; subrayan que un maestro comprometido y con vocación social puede convertirse en piedra angular para favorecer la organización de la población en la realización de diferentes proyectos comunes.

Sin embargo, y según se pudo corroborar, existen discontinuidades entre lo deseado y lo experimentado, entre el modelo tradicional del maestro rural en el imaginario de la gente y la figura del funcionario escolar en la práctica concreta. Hoy en día, son muy pocos los maestros rurales que asumen responsabilidades fuera del aula, y mucho menor el número de quienes entienden el liderazgo comunitario como una de sus tareas cotidianas. Todo parece indicar que, para la gente, el arquetipo del maestro rural se ha descolocado de su esencia deviniendo en un profesional que opera exclusivamente como enseñante de una disciplina en el interior del aula. De modo que la incompatibilidad entre las creencias de la población y lo que realmente acontece, se ha convertido en un punto de discrepancia entre la escuela y la comunidad, especialmente porque en este contexto el maestro es concebido como eje de articulación entre las dos partes 5 .

No obstante, profundizando en este hecho, se pudo reconocer que no todos los maestros en ejercicio se distancian en igual medida del modelo primigenio; de hecho, todavía persisten algunos de ellos que luchan por defenderlo y preservarlo. En lo que sigue, se describen las tipologías de maestros rurales encontradas en este sentido.

Tipología 1: maestros nativos. Se trata de maestros cuya familia y lugar de residencia permanente se encuentra en la misma comunidad donde está localizada la escuela. Algunos nacieron directamente en el pueblo y otros inmigraron con sus familias hace algunos años. Casi todos estudiaron en alguna universidad de la región y una vez obtuvieron la licenciatura gestionaron su nombramiento para ejercer en el pueblo como educadores. Usualmente son los maestros más veteranos del plantel educativo y, al mismo tiempo, el recorte menos numeroso.

\footnotetext{
5 Cabe subrayar, aun así, que la normativa que reglamenta el estatuto de la profesión docente en Colombia (Decreto 2277 de 1979, Ley General de Educación de 1994 y Decreto 1278 de 2002) en ningún momento define una distinción de funciones entre el docente urbano y el docente rural. Por consiguiente, las funciones diferenciadas para el docente rural que se destacan en esta investigación corresponden a una construcción comunitaria de base, y en ningún caso a una definición por vía legal. Sin embargo, y como se hará notar en el acápite de discusión, la literatura académica parece armonizar más con la perspectiva comunitaria que con la consigna legal.
} 
En su haber pedagógico, se caracterizan por una particular vocación de liderazgo y un alto grado de compromiso con el devenir de la escuela y de la población. Reconocen que el liderazgo comunitario constituye una de sus funciones sustantivas y son conscientes de que su trabajo debe desbordar el aula de clase para tener un impacto significativo en la vida de las comunidades. Saben que este tipo de compromiso reviste un trabajo adicional no remunerado, pero lo hacen porque encuentran una franca satisfacción contribuyendo al desarrollo de su pueblo. A esto se refieren Mayoral et al. (2018) cuando subrayan que el liderazgo de los maestros se configura alrededor del deseo experimentado por estos para transformar el medio social y educativo en el que ellos mismos crecieron.

Sin embargo, estos mismos maestros reconocen que su compromiso social y su capacidad de liderazgo se han disipado de manera progresiva en los últimos años, principalmente porque sus iniciativas no encuentran resonancia entre el grueso de la población, aunado a que no cuentan con el apoyo de algunos compañeros ni de las directivas de la institución, con quienes usualmente sostiene relaciones conflictivas, todo lo cual ha provocado que sus iniciativas se limiten a un espectro de prácticas aisladas de muy poco impacto y aceptación.

Tipología 2: maestros residentes. Son maestros provenientes de otros lugares diferentes al pueblo donde se encuentra ubicada la escuela; residen en el pueblo de lunes a viernes y se ausentan por completo en temporada de vacaciones. En su actuar, se limitan exclusivamente a cumplir con las obligaciones académicas dentro del aula, al tiempo que no evidencian ningún tipo de disposición para adjudicarse responsabilidades más allá de su jornada. A pesar de que habitan en el pueblo cinco días a la semana y de que presencian las problemáticas que existen, no se involucran demasiado en la vida de las comunidades y mucho menos se esfuerzan por proponer mecanismos de transformación, por lo que su contacto con la gente se reduce a una convivencia nominal, poco significativa.

Tipología 3: maestros forasteros. Son maestros que habitan en otros lugares diferentes al pueblo donde se encuentra ubicada la escuela. Todos los días se desplazan desde su lugar de residencia (comúnmente en motocicleta) hasta el pueblo y regresan una vez finaliza la jornada, por lo que su contacto con la comunidad es prácticamente inexistente. En sus prácticas cotidianas dilucidan un alto grado de indiferencia frente a las problemáticas que experimenta la población y no contemplan que el trabajo comunitario constituya una de sus responsabilidades.

Como se puede apreciar, los maestros residentes y forasteros (recorte más numeroso dentro del cuerpo docente) son quienes más se han distanciado del modelo original de maestro rural que alude a la tradición de la comunidad. Para ellos, en sintonía con lo descrito por Boix y Domingo (2018) sobre lo que sucede en los países en desarrollo, las condiciones de trabajo son precarias y no comportan ningún tipo de satisfacción ni recompensa, a tal grado que todos los años muchos solicitan el traslado hacia otros lugares, incrementando de esta manera el tráfico de maestros que llega cada año y que al cabo de una temporada se marcha sin dejar ningún tipo de huella. Esta situación, aludiendo a Abós y Lorenzo (2019), puede obedecer a un desajuste en los procesos de inserción e inmersión de los maestros al medio rural, quienes por falta de formación (teórica y práctica) sobre las singularidades que comporta este contexto, nunca logran adaptarse.

Así, la indiferencia social presente en la mayoría de los maestros residentes y forasteros, sumado al desencanto progresivo de los maestros nativos en lo que se refiere al trabajo comunitario, ha desatado una percepción negativa de la población frente al cuerpo docente en general. Otrora, el maestro rural era valorado como un referente social en el que se depositaban muchas de las esperanzas a nivel comunitario, incluso comportaba una adhesión particular como profesión futura entre los jóvenes, pero en la actualidad se encuentra desprovisto de ese tipo de atributos, degenerando en un objeto de críticas y cuestionamientos por parte de la comunidad en general. A este respecto, un maestro reconoce: 
La Institución hace varios años ha venido divorciada con la comunidad y la comunidad con la Institución. Por qué, no nos están viendo como las personas que estamos trabajando en beneficio de la comunidad, sino que nos están viendo como que no estamos haciendo las cosas bien, no estamos haciendo lo que nos compete, la comunidad nos pone en tela de juicio, nos está cuestionando nuestra labor permanentemente. (Ma-Pa-Ei)

En el fondo, las tensiones existentes en la comprensión de la escuela, asociadas a la imagen del maestro, no obedecen exclusivamente al comportamiento de los funcionarios de turno, sino al "descolocamiento" que está sufriendo la figura del maestro rural, respecto a su concepción tradicional aún presente en el imaginario de la gente. En su forma de enjuiciar las actuales circunstancias, los actores locales están evocando el retorno a la figura del maestro rural arquetípico, aquel que perteneció al origen de la escuela y que durante mucho tiempo trabajó por su sostenimiento, aun en medio de la clandestinidad. Aluden al maestro que se fundía en un solo cuerpo con la comunidad, que lideraba procesos sociales de diferente índole, que no se lamentaba frente a las adversidades de su lugar de trabajo y que disfrutaba del estilo de vida campesino. Se refieren a la figura del maestro rural que se ha ido diluyendo en medio del alto flujo de maestros que arriban al pueblo todos los años y que al cabo de una temporada se marchan apelando a mejores plazas de trabajo.

\section{| Discusión}

La discusión que se propone a continuación pretende mostrar cómo se articulan los resultados aquí expuestos con lo hallado por otros estudios afines en el ámbito iberoamericano; para ello se revisaron y cotejaron documentos de investigación publicados en los últimos cinco años sobre los tres ejes de comprensión, hechos notar en el presente artículo: relaciones escuela-comunidad, directores escolares y maestros rurales. Como es habitual, en algunos casos lo aquí develado encuentra una línea de continuidad con los planteamientos que reposan en la literatura, pero en otros, ofrece perspectivas complementarias que con seguridad pueden contribuir a la profundización-especificación de la teoría constituida alrededor de estos tres tópicos.

En lo tocante a la relación escuela-comunidad, la literatura disponible deja entrever dos tendencias en desarrollo; una que profundiza en el aporte que esta comunión puede hacer al mejoramiento del logro educativo de los estudiantes, asi como al buen funcionamiento de los establecimientos escolares; y otra que problematiza la reciprocidad entre las dos partes en orden a favorecer los diferentes procesos a nivel comunitario.

Efectivamente, Ortega y Cárcamo (2018) destacan la importancia que reviste la participación de las familias en la escuela como tributo al buen desempeño académico de los alumnos, al tiempo que favorece la prevención de conductas que pueden ser consideradas disruptivas para el normal desarrollo de las actividades escolares. Subrayan, además, que dicha participación debe ser promovida principalmente por medio del liderazgo de los maestros y estar orientada a la toma de decisiones institucionales. En la misma línea argumentativa, Rivera y Zavala (2019), en una revisión de seis modelos educativos diseñados para el medio rural, concluyen que la articulación con la comunidad local, tanto en la fase de diagnóstico como de diseño y ejecución, ha sido una pieza clave en el éxito de todos los modelos. 
De otro lado, Herrera (2016), a partir de un análisis a nueve experiencias en América Latina, describe la articulación que existe entre el desarrollo de base y la educación en contextos locales. Si bien visualiza que pueden existir diferentes niveles de reciprocidad entre las dos partes, concluye que habitualmente el desarrollo de base se encuentra al servicio de la educación, y no la educación al servicio del desarrollo de base y de la cualificación del capital social. En cierta congruencia con este planteamiento, pero desde una perspectiva más exhortativa que descriptiva, Ospina y Manrique (2015) reconocen que la escuela continúa plegada a una concepción convencional que la circunscribe al desarrollo académico de los estudiantes, e insisten en que se requiere transcender esta visión para que la escuela pueda contribuir también a la resolución de los problemas de su entorno y por esta vía al mejoramiento de las condiciones de vida de las comunidades, esto es, al desarrollo local.

Los resultados expuestos en el presente artículo comulgan en buena medida con lo revisado en la literatura, especificamente porque los actores locales reconocen la importancia de la reciprocidad entre las dos partes, empero, se observa una mayor adhesión a la segunda tendencia. Según se pudo apreciar, la población, en su idea original, concibe la escuela como un bien social que puede contribuir al mejoramiento de las condiciones de vida de la comunidad a través de diferentes canales, uno de ellos es la escolarización, pero también se espera que coadyuve a la preservación de las tradiciones culturales, a la cohesión y organización comunitaria, así como a la resolución de problemáticas concretas. Esto está indicando que, en contextos rurales como este (remotos y dispersos), la escuela cumple una función social, cultural y política fundamental para el desarrollo de base de las comunidades, de suerte que enclaustrarla en los procedimientos escolares exclusivamente puede socavar su potencial transformador y demorar la participación de diversos actores, con sus particulares formas de entender y relacionarse con el mundo, en los procesos de producción del conocimiento (Falla et al., 2016).

En lo relativo a la figura del director escolar, el habeas teórico de los últimos años ha girado en torno a tres discusiones, en primer lugar, la problematización acerca de las habilidades que se requieren para el ejercicio de la dirección en el ámbito escolar; en segundo lugar, la argumentación a favor de una dirección democrático-participativa que promueva la implicación de todos los actores en la cogestión de los establecimientos escolares; finalmente, la deliberación en torno a las diferentes dimensiones implicadas en la dirección escolar, más allá del plano exclusivamente administrativo.

En este orden de ideas, Barrientos et al. (2016) defienden la tesis de que los directores escolares deben disfrutar tanto de recursos técnicos y profesionales como de habilidades sociales, cualidades personales y valores, pues solo así pueden desplegar una gestión idónea y al mismo tiempo humanizadora, lo cual les implica necesariamente sustraerse de la omnipresente autoridad heredada del cargo. En continuidad con este planteamiento, Díaz (2016) reconoce al director escolar como un sujeto dotado de cualidades personales que lo constituyen en un líder frente a los demás estamentos de la comunidad, dentro de las cuales destaca la escucha activa, la sensibilidad social y la cercanía en el trato con la gente; subraya, además, que esta forma de gestión contribuye a crear una atmosfera de confianza, camaradería y corresponsabilidad necesaria para el buen funcionamiento de los centros escolares.

Desde otro ángulo, Bolívar (2019) distingue dos concepciones en el ejercicio de la dirección escolar, una burocrático-administrativa donde el director se sitúa como un representante de la administración en la escuela, y otra corporativista-profesional donde el director funge como garante y defensor de los intereses de los maestros frente a la administración. El autor reconoce la coexistencia de estas dos concepciones como algo conflictivo, por lo que propone la movilización hacia un liderazgo pedagógico que propenda por la autonomía de las escuelas e incentive la participación de todos los maestros en la definición de los proyectos escolares. 
En sintonía con esta propuesta, pero implicando también a los demás actores educativos y no sólo a los maestros, Díaz (2016) y Mayoral et al. (2018) coinciden en la importancia de una dirección escolar que no solo convoque, sino que fomente de diferentes maneras la implicación real de todos los estamentos en la dinámica de la vida escolar, por lo que se hace imperativo que su radio de actuación, además de lo administrativo, abrace también la dimensión académica, pedagógica y comunitaria. Con este propósito es fundamental que el director escolar se involucre en las prácticas de la vida local y en este sentido pueda avivar el interés de las familias por los asuntos de la escuela.

En líneas generales, los resultados expuestos en el presente artículo se ubican en un vector de continuidad con lo revisado en la literatura, no obstante, también se pueden advertir otros focos de análisis. Un hecho que se destaca en el caso estudiado es la total autonomía de la que disfrutan los directores escolares para la administración de los fondos educativos asignados a cada institución y el estatus de poder que llegan a ostentar en las comunidades producto de esta misma circunstancia. Aunado a que muchos de ellos no disfrutan de las cualidades personales hechas notar y a que en su gran mayoría provienen del casco urbano y no precisamente de las mismas comunidades, el poder que ostentan ha provocado la formación de relaciones clientelares con algunos segmentos de la población en detrimento de otros. Asimismo, el presente estudio pone de manifiesto la necesidad de definir con suficiente claridad las dimensiones que debe cubrir la dirección escolar en el medio rural, así como los requisitos de diferente índole (formación profesional, facultades humanas, visión educativa, tipo de liderazgo) que debe cumplir quién aspire a ser nombrado para este ministerio.

Finalmente, en lo que concierne al maestro rural, la literatura disponible gira en torno a dos grandes pivotes: la especificidad del magisterio en el medio rural y los procesos de formación inicial y permanente del profesorado. En efecto, Zamora y Mendoza (2018) definen cinco notas distintivas en la gramática del maestro rural: vinculación con la comunidad, didácticas del aula multigrado, adaptación a modelos flexibles, dilema entre el arraigo o desarraigo de los jóvenes y creatividad para encarar las condiciones del medio. En consonancia con ese planteamiento, Brumat y Baca (2015), a partir de un estudio sobre prácticas docentes en contextos de ruralidad, describen como funciones sustantivas de estos profesionales la dirección de los centros, el cuidado de las instalaciones y el liderazgo comunitario.

De otro lado, Abós y Lorenzo (2019) sostienen la tesis de que las dificultades experimentadas por los maestros rurales yacen principalmente en la escasa o casi nula preparación que estos reciben para desempeñarse en este contexto, por lo que proponen la incorporación de formación teórica y práctica, desde y para lo rural, que favorezca los procesos de inducción e inmersión de los profesionales a este escenario particular. En su dimensión descriptiva, este esbozo sintoniza con Zamora y Mendoza (2018) cuando problematizan que, en Colombia, sólo el $11 \%$ de las Escuelas Normales Superiores y el 6 \% de las Facultades de Educación (escenarios de formación de maestros) tienen incluido el componente rural como parte de su currículo.

En un plano argumentativo complementario, Juárez y Rodríguez (2016) y Weiss (2017) dejan entrever que la alta fluctuación de docentes en el medio rural obedece, por un lado, a la imposibilidad de retener a los licenciados mejor preparados y de mayor experiencia por cuenta de las precarias condiciones de trabajo y a la dispersión de las escuelas; por otro lado, a la preeminencia de los criterios políticos sobre los pedagógicos-vocacionales en la selección de los docentes destinados a este contexto. En la misma dirección, pero desde una óptica intercontinental, Boix y Domingo (2018) sostienen que si bien en los países en desarrollo los maestros rurales experimental limitaciones de diferente naturaleza para el ejercicio de sus funciones, en "países desarrollados están altamente formados y capacitados, ejercen en edificios estables y seguros, con buena iluminación, disponen de una gran variedad de recursos didácticos y sus sustitutos son también maestros" (p. 6). 
Algunos de los planteamientos presentes en la literatura puede que estén a la base de los resultados expuestos; es muy probable, por ejemplo, que el descrédito que sufre el maestro en el caso estudiado obedezca a incongruencias en los procesos de formación y selección del profesorado. Empero, lo que el presente artículo quiere hacer notar con mayor insistencia, es la discontinuidad que se produce entre la imagen arquetípica del maestro, aún presente en el imaginario de la gente, y la forma como los actuales maestros entienden y desarrollan sus funciones. No es un asunto menor que los actores locales valoren al maestro no tanto por el logro académico de los estudiantes (algo que en otros contextos sería suficiente), sino por su grado de implicación en los asuntos comunitarios, la contribución a la resolución de problemáticas concretas y el nivel de adaptación al estilo de vida campesino. En esta línea, se subraya la importancia de diferenciar el estatuto del maestro rural (nombramiento, incentivos, funciones, escalafonamiento, evaluación) dentro de la normativa oficial, por cuanto las implicaciones y demandas de su sitio de trabajo no le permiten homologarse en muchos aspectos con el maestro urbano, y es precisamente esta falta de especificación en la trastienda legal la que frecuentemente origina problemas de comprensión.

\section{| Conclusiones}

La manera como los actores rurales comprenden el sentido de la escuela en el medio rural está directamente asociada a las concepciones sobre la escuela que se remontan al origen de los centros y que se traducen en imágenes arquetípicas sobre las relaciones, los directores y los maestros. Son comprensiones que se han ido consolidando en la línea de las tradiciones comunitarias y configurando en armonía con la formación de su repertorio axiológico endógeno. Es por eso que frecuentemente las medidas de optimización del servicio educativo que se piensan exclusivamente desde otras instancias (centralizadas y omnicomprensivas), si bien abrazan un aparente progreso en términos formales (cobertura), en la práctica terminan desatando dinámicas comunitarias antagónicas que problematizan la credibilidad y el funcionamiento mismo de la escuela, provocando así tensiones y discontinuidades en la manera como los actores rurales significan su propia realidad social y educativa.

Igual consideración merece la forma como las comunidades locales (rurales y remotas principalmente), experimentan el surgimiento y evolución de las transiciones a la democracia en la región y más específicamente los resultados de los procesos de descentralización. La certificación administrativa de los municipios y con ello la transferencia de responsabilidades y recursos para atender las necesidades del sector educativo, al privilegiar una mirada técnico-administrativa que obvia la semantización comunitaria de la escuela, conduce a desajustes incluso más perjudiciales que las mismas privaciones materiales.

Pocos podrían objetar que acciones como la asignación de fondos educativos, la designación de directores con facultades administrativas y el incremento de maestros, pueden conducir a una cualificación de los centros escolares que operan en el medio rural; no obstante, soslayar las comprensiones históricas sobre la escuela que poseen los actores locales y la manera como ellos valoran esas mismas acciones, es lo que puede conllevar a los antagonismos anteriormente mencionados. En municipios como La Pascuala, Tacaloa y Coyongal, los actores locales valoran el incremento de los fondos educativos como algo necesario, pero insisten en que se requiere un modelo de administración mucho más democrático, de suerte que los recursos surtan los efectos deseados y no se conviertan en un elemento que desata todo tipo de disputas, conflictos y rivalidades. 
La designación de directores mejor preparados a nivel administrativo puede conducir a una gestión más eficiente de los centros escolares, pero para que la escuela cumpla la función social que le es propia y no se pliegue llanamente a los procesos de escolarización, se requiere clarificar desde el inicio que los directores deben disfrutar también de otras cualidades, como capacidad de liderazgo, sensibilidad social y disponibilidad para trabajar de la mano con la gente. El incremento de la planta docente constituye uno de los aspectos medulares en orden a ofrecer una educación de mejor calidad en el medio rural; sin embargo, la sola elevación del número no es suficiente, además, es de vital importancia garantizar que los maestros nombrados para ejercer en el sector disfruten de otras facultades, como apertura para fungir de vehículo entre la escuela y la comunidad, disposición para trabajar en el medio rural de manera continua y no sólo transitoria, y vocación para asumir el liderazgo comunitario como una de sus funciones sustantivas.

\section{| Referencias}

Abós, P., y Lorenzo, J. (2019). Ser maestro en la escuela rural, de la obligación a la opción. Cuadernos de Pedagogía, (496), 77-83

Ariza, N., Quiroga, B., y Ardila, M. (2017). Revisión de gasto sector educación. Fedesarrollo.

Asela, J. (2015). La descentralización en Colombia, ¿realidad o espejismo? Consideraciones sobre la actualidad del proceso. Revista CEA, 1(1), 65-74. https://doi.org/10.22430/24223182.63

Barrientos, C., Silva P., y Antúnez, S. (2016). Competencias directivas para promover la participación: familias en las escuelas básicas. Revista Educación, 25(49), 45-62. https://doi.org/10.18800/educacion.201602.003

Boix, R., y Domingo, L. (2018). La escuela rural, una mirada intercontinental. Aula de Innovación Educativa, (270), 14-27.

Bolivar, A. (2019). Políticas de gestión escolar desde una perspectiva comparada: la excepción ibérica. Economía de la Educación y Política Educativa, (910), 93-104.

Braga, M., y Acuña, I. (2015). El fortalecimiento de la democracia en Brasil y los retos de la observación electoral. Revista América Latina Hoy, (70), 91-108. http://dx.doi.org/10.14201/alh20157091108

Brumat, M., y Baca, C. (2015). Prácticas docentes en contextos de ruralidad. Un estudio en escuelas rurales del norte de Córdoba. Educación, Formación e Investigación, 1(2), 1-16.

Castro, M. (2007). La escuela en la comunidad. La comunidad en la escuela. Graó.

Codoceo, F., y Muñoz, J. (2017). Fragmentación, asistencialismo e individualización de la responsabilidad: perspectivas territoriales concernientes a la percepción ciudadana de las políticas públicas en el contexto chileno de producción neoliberal. Revista Sociedade e Estado, 32(2), 371-387. http://doi.org/10.1590/s0102-69922017.3202005

Congreso de la República de Colombia. (1994). Ley 115, por la cual se expide la Ley General de Educación.

Congreso de la República de Colombia. (2001). Ley 715, por la cual se dictan normas orgánicas en materia de recursos y competencias de conformidad con los artículos 151, 288, 356 y 357 (Acto Legislativo 01 de 2001) de la Constitución Política y se dictan otras disposiciones para organizar la prestación de los servicios de educación y salud, entre otros.

Díaz, E. (2016). Liderazgo, comunicación y trabajo en equipo: la tríada inseparable de una gestión escolar integral. Dilemas Contemporáneos: Educación, Política y Valores, 4(2), 1-19.

Díaz, E., y Paillama, D. (2017). Clientelismo y corrupción en contextos de baja estatalidad, una relación mutualista. Revista de Sociología e Política, 25(64), 73-98.

http://doi.org/10.1590/1678-987317256406 
Durston, J., Gaete, J., y Pérez, M. (2016). Comunidad, conectividad y movimiento regional en la Patagonia: evolución del capital social en la región de Aysén, Chile. Revista CEPAL, (116), 235-249. http://hdl.handle.net/11362/40040

Falla, S., Hermelin, D., y Aguirre, C. (2016). Conectar comunidades para construir sentidos sociales en torno al conocimiento. Trilogía Ciencia Tecnología Sociedad, 8(15), 57-68.

https://doi.org/10.22430/21457778.400

Geertz, C. (1992). La interpretación de las culturas. Gedisa.

González, M., y Liendo, N. (2017). La defensa colectiva de la democracia en América Latina: ¿por qué?, ¿cómo?, ¿cuándo? Análisis Político, (91), 3-17. https://doi.org/10.15446/anpol.v31n91.70261

Guber, R. (2016). La etnografía: método, campo y reflexividad. Siglo XXI.

Hammersley, M. (2016). Reading ethnographic research. A critical guide. Taylor \& Francis

Herrera, J. (2016). La relación escuela-comunidad: un análisis de la teoría de sistemas a nueve experiencias de América Latina. Revista Interamericana de Educación, Pedagogía y Estudios Culturales, 9(1), 11-33.

Ibáñez, J. (1992). Más allá de la sociología: el grupo de discusión, técnica y crítica. Siglo XXI.

Jiménez, W. (2019). Características, aportes y tendencias del constitucionalismo colombiano en 200 años de andadura. Diálogos de Saberes, (51), 99-116.

https://doi.org/10.18041/0124-0021/dialogos.51.2019.5870

Juárez, D., y Rodríguez, C. (2016). Factores que afectan la equidad educativa en escuelas rurales de México. Pensamiento educativo, 53(2), 1-15. http://doi.org/10.7764/PEL.53.2.2016.8

Machado. A. (2017). El problema de la tierra: conflicto y desarrollo en Colombia. Debate.

Martínez, L. (2019). Clientelismo en los agronegocios de Ecuador: empresarios y trabajadores rurales. Revista Europea de Estudios Latinoamericanos y del Caribe, (107), 75-94. http://doi.org/10.32992/erlacs.10415

Mayoral, D., Colom, J., Bernad, O., y Torres T. (2018). Liderazgo en la Escuela Rural: Estudios de Casos. International Journal of Sociology of Education, 7(1), 49-70. http://doi.org/10.17583/rise.2018.2637

Ministerio de Educación Nacional -MEN-. (2017). Plan Especial de Educación Rural. MEN. https://www.mineducacion.gov.co/1759/articles-385568_recurso_1.pdf

Ogbu, J. (2005). Etnografía escolar: una aproximación a nivel múltiple. En M. Velasco, F. García, y A. Díaz de Rada (Eds.), Lecturas de antropología para educadores (pp. 145-174). Trotta.

Ortega, D., y Cárcamo, H. (2018). Relación familia-escuela en el contexto rural. Miradas desde las familias. Revista Educación, 27(52), 98-118. https://doi.org/10.18800/educacion.201801.006

Ospina, M., y Manrique, D. (2015). El reto de la escuela: profundizar su relación con la comunidad, Zona Próxima, (22), 236-249. http://dx.doi.org/10.14482/zp.22.6094

Pérez-Liñán, A., y Mainwaring, S. (2015). La supervivencia de la democracia en América Latina (1945-2005). Revista América Latina Hoy, (68), 139-168. http://dx.doi.org/10.14201/alh201468139168

Presidencia de la República de Colombia. (1979). Decreto 2277, por el cual se adoptan normas sobre el ejercicio de la profesión docente.

Presidencia de la República de Colombia. (2002). Decreto 1278, por el cual se expide el estatuto de profesionalización docente.

Rivera, A., y Zavala, M. (2019). Construcción de alternativas a problemáticas sentidas. Revisión de modelos educativos para el medio rural, Revista Latinoamericana de Educación y Estudios Interculturales, 3(4), 70-86

Rodriguez, F. (2017). Reflexiones sobre el concepto cultura política y la investigación histórica de la democracia en América Latina. Historia y Memoria, (14), 205-247.

http://dx.doi.org/10.19053/20275137.n14.2017.5820

Saelzer, G., Soto, M., y Álvarez, L. (2019). Condiciones de desarrollo en espacios patrimoniales y remotos. Caso de planificación de Río Serrano, comuna Torres de Payne, Chile. Revista de Geografia Norte Grande, (72), 93-112. http://dx.doi.org/10.4067/S0718-34022019000100093 
Sautu, R. (2015). Requisitos formales teórico-metodológicos para la construcción del marco teórico de una investigación en Ciencias Sociales. Revista Latinoamericana de Metodología de la Investigación Social, (10), 7-20.

Schütz, A. (1995). El problema de la realidad social. Amorrortu

Sili, M. (2019). Acción territorial y construcción del desarrollo. La experiencia de zonas rurales de la Pampa argentina. Revista de Geografia Norte Grande, (74), 159-186. http://dx.doi.org/10.4067/S0718-34022019000300159

Weiss, E. (2017). Estudio exploratorio del modelo de telebachillerato comunitario y su operación en los estados. INEE.

Wolcott, H. (2005). El maestro como enemigo. En Velasco, M. García, F. y Díaz de Rada, A. (Eds.), Lecturas de antropología para educadores (pp. 243-258). Trotta.

Wolcott, H. (2016). Ethnography lessons. A primer. Taylor \& Francis.

Zamora, L., y Mendoza, A. (2018). La formación de educadores para el trabajo rural: el reto planteado por la escuela rural multigrado en Colombia. Nodos y Nudos, 6(45), 72-85.

Zúñiga, L., y Valencia, H. (2016). Ciudadanía y democracia en Cartagena: entre la exclusión y el clientelismo político. Reflexión Política, 18(36), 144-157. https://doi.org/10.29375/01240781.2658 